\title{
Acute Ulcerative Colitis, Thrombocytopenia, and Venous Thromboembolism Treated With Combined Laparoscopic Splenectomy and Colectomy
}

\author{
Ahmed Dehal, MD, MPH, Sunal Patel, MD, Samir Johna, MD, Patrick Nguyen, MD \\ Department of General Surgery, Kaiser Permanente, Fontana, CA, USA (all authors).
}

\begin{abstract}
Immune thrombocytopenic purpura and venous thromboembolism are rare but known complications of ulcerative colitis. Although several case studies have examined the treatment options available for ulcerative colitis patients presenting with immune thrombocytopenic purpura, there have been no reported cases that describe the optimal treatment for patients with ulcerative colitis and immune thrombocytopenic purpura complicated by simultaneous deep venous thromboses. We present the case of a 19-year-old woman who presented with ulcerative colitis and immune thrombocytopenic purpura and in whom multiple deep venous thromboses also developed. The patient underwent urgent simultaneous laparoscopic colectomy and splenectomy. She improved clinically, and her platelet count recovered after surgery. When thrombocytopenia develops in patients with ulcerative colitis, a diagnosis of immune thrombocytopenic purpura should be considered. In such patients, pre-existing ulcerative colitis might be involved in the immunologic causal mechanism of immune thrombocytopenic purpura and venous thromboembolism. In cases in which these entities are refractory to medical management or complicated by venous thromboembolism, simultaneous laparoscopic colectomy and splenectomy are safe and well tolerated and can be lifesaving.
\end{abstract}

Key Words: Ulcerative colitis, Thrombocytopenia, Deep venous thrombosis, Colectomy, Splenectomy

Citation Dehal A, Patel S, Johna S, Nguyen P. Acute ulcerative colitis, thrombocytopenia, and venous thromboembolism treated with combined laparoscopic splenectomy and colectomy. CRSLS e2014.00298. DOI: 10.4293/CRSLS.2014.00298.

Copyright (C) 2014 SLS This is an open-access article distributed under the terms of the Creative Commons Attribution-Noncommercial-ShareAlike 3.0 Unported license, which permits unrestricted noncommercial use, distribution, and reproduction in any medium, provided the original author and source are credited.

Address correspondence to: Ahmed Dehal, MD, MPH, Department of General Surgery, Kaiser Permanente, 9961 Sierra Ave, Fontana, CA, USA. Zip Code: 92335 Telephone: (912) 427-5000, E-mail: ahmed.dehal@gmail.com

\section{INTRODUCTION}

Ulcerative colitis (UC) is a chronic inflammatory bowel disease (IBD) of unknown etiology. There are several extraintestinal manifestations of IBD that have been reported in the literature. Several autoimmune conditions have been shown to be associated with IBD, among which autoimmune hemolytic anemia is frequently reported. ${ }^{1,2}$ Immune thrombocytopenic purpura (ITP) has been reported sporadically in the literature. ITP is a type of thrombocytopenia that is defined by platelet destruction as a result of autoantibodies to platelet antigens. Edwards and Truelove ${ }^{3}$ originally described the association between ITP and UC in 1964. Another rare extraintestinal manifestation of IBD is venous thromboembolism (VTE). UC, in particular, has been shown to have higher coagulative-fibrinolytic activity that is thought to account for the increased risk of VTE seen in this patient population. ${ }^{4}$
We present the case of a 19-year-old patient with both UC and ITP. Interestingly, however, multiple deep venous thromboses (DVTs) also developed and complicated her treatment. Although several case studies have examined the treatment options available for UC presenting with ITP, there have been no published cases that describe the management of patients with UC and ITP complicated by simultaneous DVTs.

\section{CASE REPORT}

A 19-year-old woman with a history of UC was admitted to the hospital because of hematochezia and abdominal pain. Physical examination findings showed abdominal tenderness and tachycardia. The laboratory workup showed an elevated white blood cell count of $12900 / \mathrm{mm}^{3}$ and a normal platelet count of $171000 / \mathrm{mm}^{3}$. Soon after admission, a trend for thrombocytopenia $\left(119000 / \mathrm{mm}^{3}\right)$ 
was noted. Computed tomography scan of the abdomen showed diffuse colonic thickening. Flexible sigmoidoscopy showed severe colitis and evidence of nonspecific colitis on biopsy. A diagnosis of acute UC flare-up was established based on the patient's clinical features and colonoscopic appearance. She was given total parenteral nutrition, started taking oral 5-aminosalicylic acid (balsalazide) in addition to prednisolone, and underwent steroid pulse therapy. The patient showed no response to treatment and continued to have worsening hematochezia, and the platelet count continued to fall during the hospitalization course. The administration of balsalazide was stopped because it was suspected as a possible cause of thrombocytopenia, but the platelet count continued to decrease and reached $64000 / \mathrm{mm}^{3} 7$ days later. Disseminated intravascular coagulation was ruled out by the presence of a normal prothrombin time, as well as normal levels of fibrinogen and fibrin degradation products. A diagnosis of ITP was suspected based on the finding of positive autoantibodies to platelet membrane antigen and the absence of splenomegaly. Intravenous $\gamma$-globulin was administered and the platelet count rose transiently, but exacerbation of the UC continued. Severe anemia developed due to intractable hematochezia, and the patient required several transfusions of packed red blood cells. More aggressive alternative therapy such as infliximab (Remicade: Janssen Biotech, Inc. Titusville, NJ, USA), adalimumab (Humira: Abbvie Inc. North Chicago, Illinois, USA), and cyclosporine could not be started because of the severe thrombocytopenia. The blood platelet count continued to decrease significantly to as low as 4000/ $\mathrm{mm}^{3}$, and the patient continued to have severe hematochezia. Meanwhile, left leg and right arm DVTs developed. Anticoagulation could not be started because of bleeding. Surgical treatment was considered necessary because of the life-threatening nature of the disease. The patient underwent combined laparoscopic splenectomy and total colectomy. Postoperatively, she improved and the platelet count normalized $\left(210000 / \mathrm{mm}^{3}\right)$ within 2 days.

\section{Surgical Technique}

Under general anesthesia, the patient was placed in the lithotomy position and was prepared and draped in the usual sterile fashion. A 5-mm supraumbilical incision was made, a Veress needle was inserted, and pneumoperitoneum was established. A 5-mm trocar was placed, and a 5-mm, $30^{\circ}$ laparoscope was then placed into the abdomen. Four more trocars were placed under direct vision: two 5-mm trocars were placed in the epigastric area and the suprapubic position, respectively; a $12-\mathrm{mm}$ trocar was placed through the previously marked ileostomy site in the left lower part of the abdomen; and a $12-\mathrm{mm}$ trocar was placed in the right part of the mid abdomen. We then started the procedure by mobilizing the splenic flexure, the hepatic flexure, the right part of the colon, and then the left part of the colon using the lateral-to-medial approach. Complete mobilization of the sigmoid colon was performed to slightly below the level of the peritoneal reflection. We then transected the upper rectum with an Endo GIA (Covidien, Mansfield, MA, USA) stapler.

After the colon was distally transected, it was left in the abdominal cavity and attention was then paid to the spleen. Mobilization of the spleen by dividing the 4 major splenic ligaments was completed. The major vascular supply to the spleen was taken down by use of the vascular stapler. After the spleen was completely mobilized and detached from its blood supply, an Endocatch (Covidien, Mansfield, MA, USA) bag was introduced and the spleen was extracted through the left lower 12-mm port. Next, the colon was transected at the ileocecal junction with a GIA stapler (Covidien, Mansfield, MA, USA) and extracted through the same left lower port. The distal ileum was then brought up as an ileostomy using the left lower port.

\section{DISCUSSION}

The association of ITP with UC has been well described in the literature, with a prevalence of $0.1 \%$ to $0.5 \% .{ }^{5} \mathrm{~A}$ summary of reported cases of UC and ITP in the literature ${ }^{6-24}$ is presented in Table 1. On the basis of our review, no previous cases underwent simultaneous splenectomy and colectomy. In some patients, ITP and UC were successfully treated with medical therapy consisting of short bursts of steroids and/or intravenous $\gamma$-globulin. In cases refractory to medical management, ITP has often required either splenectomy or colectomy for cure; in contrast, in a few other cases, resolution of ITP was refractory to both medical treatment and splenectomy, and in these cases, colectomy has actually resolved both UC and ITP.

The pathogenesis of the development of ITP related to UC is unclear; however, with the resolution of ITP often observed after colectomy, a colon-derived pathogenesis is plausible. One theory suggests that during severe colitis, antigens found in the colonic lumen could generate a humoral response. ${ }^{14}$ This would lead to antibody production that could potentially cross react with platelet surface antigens, thereby causing ITP. Another hypothesis in- 
Table 1.

Reported Cases of Patients With UC and Acute ITP

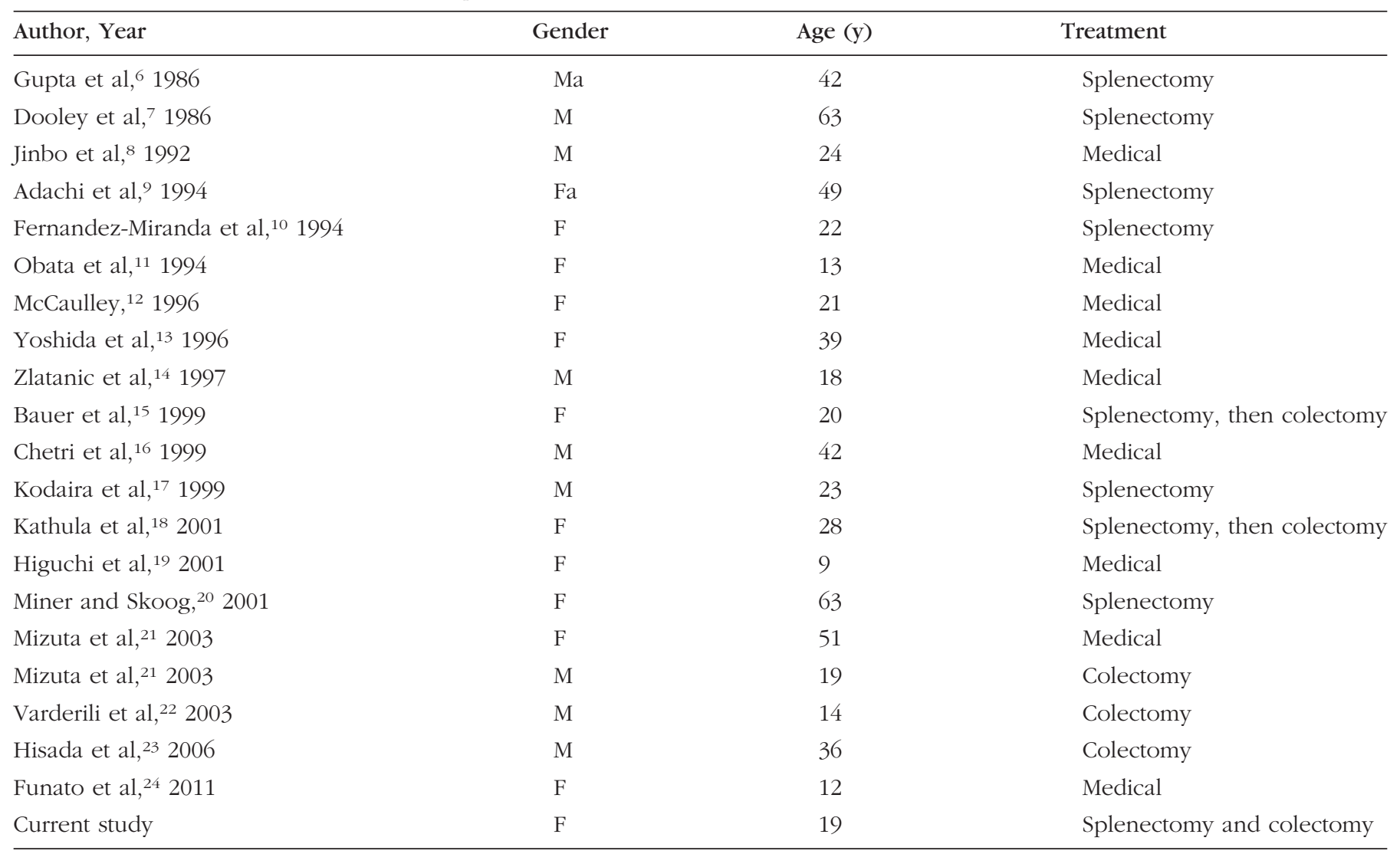

${ }^{a} \mathrm{~F}=$ female; $\mathrm{M}=$ male.

cludes the idea that during severe colitis, inflammation will lead to local destruction and sequestration of platelets, which would lead to the absence of circulating platelets. ${ }^{17}$

Several studies have reported that an increased risk of VTE exists in patients with IBD. ${ }^{25-28}$ The risk of VTE developing with IBD varies in the literature. A study by Grainge et $\mathrm{al}^{26}$ reported an 8.4 times greater risk of VTE with active IBD. In a prospective study from Japan, Sonoda et al ${ }^{27}$ found that $17 \%$ of a consecutive series of 47 patients with IBD had detectable VTE. A study from the Mayo Clinic reported that $1.3 \%$ of 7199 IBD patients had thromboembolic events. ${ }^{28}$ Of course, those patients hospitalized for their IBD were at a higher risk of VTE developing compared with ambulatory IBD patients. Previous studies showed that patients with IBD exhibited heightened coagulative-fibrinolytic activities, especially in those with UC. ${ }^{3}$ This heightened activity can explain the higher incidence of VTE development in these patients. Thus most previous reports agree that management of the inflammation associated with IBD with steroids is the best measure for the prevention of VTE. To our knowledge, the occurrence of ITP and VTE simultaneously in patients with an acute flare-up of UC has not been previously described in the literature.

Drug-related thrombocytopenia must always be considered and ruled out in UC patients before the diagnosis of ITP is established. Particularly applicable to UC patients are 5-Aminosalicylic Acid products, which have been reported to cause thrombocytopenia by direct myelosuppression. $^{29}$ Our patient did receive balsalazide (Colazal: Salix Pharmaceuticals Inc, Raleigh, NC, USA), which could potentially cause drug-related thrombocytopenia. However, this possibility was ruled out as a potential cause of the patient's low platelet count because she was thrombocytopenic before the initiation of Colazol therapy and her thrombocytopenia did not resolve after discontinuation of Colazol. 
The second line of treatment for steroid-refractory UC is a more aggressive drug such as infliximab (Remicade), adalimumab (Humira), or cyclosporine. However, because thrombocytopenia is a known side effect of these medications, we could not use them in this particular case. As medical therapy was under way for the treatment of both ITP and acute colitis, DVTs began developing in our patient, for which she required treatment as well. With platelet counts at low levels and progressively worsening colitis with active bleeding, anticoagulation treatment for the DVTs was not a viable option, and as such, the decision to abandon medical treatment and proceed with urgent surgery was made.

The decision to proceed with splenectomy and colectomy simultaneously in this case was made for several reasons. First, it is important to understand that urgent definitive treatment was critical in this case because the patient did not respond to the medical treatment and continued to have severe hematochezia and worsening anemia. Furthermore, because of the development of DVTs, the subsequent risk of pulmonary embolism without anticoagulation was high. Therefore urgent and potentially lifesaving treatment was imperative. Second, although there have been some reported cases in which splenectomy alone resolved ITP, there are other case reports in the literature that describe patients in whom ITP was refractory to splenectomy and subsequent colectomy was required (Table 1). Conversely, although colectomy alone has in some instances resolved ITP, there have also been documented cases of ITP developed after colectomy. ${ }^{30}$ As such, the only method to ensure resolution of ITP and allow for the most immediate anticoagulation therapy for the patient's DVTs was simultaneous splenectomy and colectomy.

The safety and effectiveness of both laparoscopic splenectomy and colectomy have been previously documented in the literature. Both approaches are associated with less surgical trauma and intraoperative blood loss, reduced postoperative pain, an earlier return to normal activity, improved cosmesis, and lower hospital costs compared with an open approach.31,32 Therefore, keeping these findings in mind and considering the anticipated increased risk of bleeding from the thrombocytopenia in our patient, we made the decision to proceed with the laparoscopic approach and the patient underwent combined laparoscopic splenectomy and total colectomy. She tolerated the procedure well and recovered from ITP and UC postoperatively, and she was then able to be appropriately treated for her DVTs.
In conclusion, we have presented one of the many rare occurrences of UC presenting with ITP. However, this is the first case that has been reported in which DVTsanother known but rare complication of this disease-also developed in the patient. The development of 2 DVTs in our patient complicated the treatment plan and increased the urgency for definitive treatment. As such, medical management was abandoned, and combined splenectomy and total colectomy were performed laparoscopically to manage both the UC and ITP at once. The laparoscopic approach was optimal for this patient and resulted in a satisfactory outcome. With the handful of case reports and series available, it is important to consider ITP as a potential cause of thrombocytopenia associated with acute exacerbations of UC. In cases in which these entities are refractory to medical management or complicated by occurrences of VTE, simultaneous colectomy and splenectomy may cure both UC and ITP. The laparoscopic approach is safe, feasible, and well tolerated in such patients.

\section{References:}

1. Greenstein AJ, Janowitz HD, Sachar DB. The extraintestinal complications of Crohn's disease and ulcerative colitis: a study of 700 patients. Medicine. 1976;55:401-412.

2. Gumaste V, Greenstein AJ, Meyers R, Sachar DB. Coombspositive autoimmune hemolytic anemia in ulcerative colitis. Dig Dis Sci. 1989;34:1457-1461.

3. Edwards FC, Truelove SC. The course and prognosis of ulcerative colitis. Part III: complications. Gut. 1964;5:1-15.

4. Biancone L, Scopinaro F, Maletta M, et al. Circulating D dimer in inflammatory bowel disease. Ital J Gastroenterol. 1994; 3:116-120.

5. Snook JA, de Silva HJ, Jewell DP. The association of autoimmune disorders with inflammatory bowel disease. $Q \mathrm{~J} \mathrm{Med.}$ 1989;72:835-840.

6. Gupta S, Saverymuttu SH, Marsh JC, et al. Immune thrombocytopenic purpura, neutropenia and sclerosing cholangitis associated with ulcerative colitis in an adult. Clin Lab Haematol. 1986;8:67-69.

7. Dooley DP, Mills GM, Spiva DA. Immune thrombocytopenia and ulcerative colitis. South Med J. 1986;79:1044-1045.

8. Jinbo T, Tamura J, Shinohara M, et al. Possible causal association between ulcerative colitis and idiopathic thrombocytopenia purpura. Clin Rheumatol. 1992;11:408-409.

9. Adachi Y, Nouchi T, Aoki M, et al. A case of primary sclerosing cholangitis associated with ulcerative colitis and idiopathic thrombocytopenic purpura. Jpn J Gastroenterol. 1994;91: $2278-2282$. 
10. Fernandez-Miranda C, Mateo S, Kessler P, Gonzalez-Castelló J. Immune thrombocytopenia and ulcerative colitis. J Clin Gastroenterol. 1994;18:85-86.

11. Obata S, Higashi K, Kawano F, et al. A case of Turner's syndrome with idiopathic thrombocytopenic purpura, and Hashimoto's thyroiditis detected in the course of ulcerative colitis. Jpn J Gastroenterol. 1994;91:899-903.

12. McCaulley M. Immune thrombocytopenia and ulcerative colitis: response to nonoperative therapy. J Clin Gastroenterol. 1996;23:242-243.

13. Yoshida EM, Chaun M, Freeman HJ, et al. Immune thrombocytopenic purpura in three patients with pre-existing ulcerative colitis. Am J Gastroenterol. 1996;91:1232-1235.

14. Zlatanic J, Korelitz BI, Wisch N, et al. Inflammatory bowel disease and immune thrombocytopenic purpura: is there a correlation? Am J Gastroenterol. 1997;92:2285-2288.

15. Bauer WM, Litchtin A, Lashner BA. Can colectomy cure immune thrombocytopenic purpura in a patient with ulcerative colitis? Dig Dis Sci. 1999;44:2330-2333.

16. Chetri K, Aggarwal R, Sharma B, et al. Ulcerative colitis and immune thrombocytopenia: a report of two cases. Indian J Gastroenterol. 1999;18:174.

17. Kodaira M, Hanai H, Kajimura M, et al. Further evidence that exacerbation of ulcerative colitis causes the onset of immune thrombocytopenia: a clinical case. Am J Gastroenterol. 1999;94:1408-1410.

18. Kathula SK, Polenakovik H, el-Tarabily M, Polenakovik S. Complete resolution of refractory immune thrombocytopenic purpura after colectomy for ulcerative colitis. Int J Clin Pract. 2001;55:647-648.

19. Higuchi LM, Joffe S, Neufeld EJ, et al. Inflammatory bowel disease associated with immune thrombocytopenic purpura in children. J Pediatr Gastroenterol Nutr. 2001;33:582-587.

20. Miner RJ, Skoog SM. Immune thrombocytopenic purpura, profuse diarrhea, and new onset ulcerative colitis: a dramatic response to splenectomy. J Clin Gastroenterol. 2001;32:276277.
21. Mizuta $\mathrm{Y}$, Isomoto $\mathrm{H}$, Kadokawa $\mathrm{Y}$, et al. Immune thrombocytopenic purpura in patients with ulcerative colitis. J Gastroenterol. 2003;38:884-890.

22. Varderili E, Sariçam T, Aslan V, Gülbaş Z. Resolution of immune thrombocytopenic purpura after colectomy for ulcerative colitis. Turk J Gastroenterol 2003;14:270-272.

23. Hisada T, Miyamae Y, Mizuide M, et al. Acute thrombocytopenia associated with preexisting ulcerative colitis successfully treated with colectomy. Intern Med. 2006;45:87-91.

24. Funato M, Fukao T, Sasa H, et al. Successful treatment of pediatric immune thrombocytopenic purpura associated with ulcerative colitis. Pediatr Int. 2011;5:771-773.

25. Zoller B, Li X, Sundquist J. Autoimmune diseases and venous thromboembolism: a review of the literature. Am J Cardiovasc Dis. 2012;2:171-183.

26. Grainge MJ, West J, Card TR. Venous thromboembolism during active disease and remission in inflammatory bowel disease: a cohort study. Lancet. 2010;375:657-663.

27. Sonoda K, Ikeda S, Mizuta Y, et al. Evaluation of venous thromboembolism and coagulation-fibrinolysis markers in Japanese patients with inflammatory bowel disease. J Gastroenterol. 2004;39:948-954.

28. Talbot RW, Heppell J, Dozois RR, Beart RW Jr. Vascular complications of inflammatory bowel disease. Mayo Clin Proc. 1986;61:140-145.

29. Daneshmend TK. Mesalamine-associated thrombocytopenia. Lancet. 1991;337:1297-1298.

30. Yong S, Dower N, El-Matary W. Idiopathic thrombocytopenic purpura after colectomy for pediatric ulcerative colitis. Inflamm Bowel Dis. 2008;14:1313-1315.

31. Walsh RM, Heniford BT, Brody F, Ponsky J. The ascendance of laparoscopic splenectomy. Am Surg. 2001;67:48-53.

32. Muckleroy SK, Ratzer ER, Fenoglio ME. Laparoscopic colon surgery for benign disease: a comparison to open surgery. JSLS. 1999;3:33-37. 